\title{
Los buenos profesores en la mirada de padres y apoderados
}

\section{Samuel Pérez Norambuena}

orcid.org/0000-0002-1710-328X

Universidad del Bío-Bío, Chile

sperez@ubiobio.cl

\section{Luis Linzmayer Gutiérrez}

orcid.org/00oo-0002-8687-4459

Universidad del Bío-Bío, Chile

llinzmayer@ubiobio.cl

\section{Resumen}

El objetivo del presente estudio es comprender qué significa ser un "buen profesor" para los padres y apoderados en un colegio vulnerable de Santiago, a partir de la identificación y descripción de las características de este actor educativo relevante. De una metodología cualitativa de análisis de los discursos, con enfoque fenomenológico, se dedujo que, a la hora de conceptualizar y caracterizar a un buen docente, los padres valoran la dimensión humana de este, con componentes afectivos que le permiten relacionarse empáticamente con los otros, lo que genera confianza y da apoyo formativo en la crianza de los hijos. Los padres buscan estar tranquilos respecto del presente y futuro de sus hijos y para ello necesitan la presencia de buenos profesores.

\section{Palabras clave (fuente: tesauro de la UNESCO).}

Calidad de la educación; competencias del docente; desempeño docente; evaluación de la educación; evaluación docente; familia; educación.

Recepción: 30/05/2018 | Envío a pares: 11/08/2018 | Aceptación por pares: 25/08/2018 | Aprobación: 15/11/2018 


\title{
Good Teachers. A View from Parents and Guardians
}

\begin{abstract}
The aim of this study is to understand what it means to be a "good teacher" for parents and guardians in a vulnerable school in Santiago de Chile (Chile), from the identification and description of the characteristics of this relevant educational actor. A qualitative methodology with a phenomenological approach was used to analyze the discourses of 20 families. Based on in-depth interviews and a discussion group, when it comes to conceptualizing and characterizing a good teacher, parents value their human dimension. This should be complemented with professional and affective components that allow for empathic relationships with others, building trust and formative support in upbringing their children. Parents seek to feel at ease about the present and future of their children and, for this, they need the presence of good teachers who balance human and professional aspects in the act of educating.
\end{abstract}

\section{Keywords (Source: UnEsco Thesaurus)}

Educational quality; teacher qualifications; teaching performance; educational evaluation; teacher evaluation; family; education. 


\section{Os bons professores. Um olhar a partir dos pais e responsáveis legais}

\section{Resumo}

O objetivo do presente estudo é compreender o que significa ser um "bom professor" para os pais e responsáveis legais em um colégio vulnerável em Santiago do Chile (Chile) a partir da identificação e descrição das características desse agente educativo relevante. Utilizou-se uma metodologia qualitativa de análise dos discursos, com enfoque fenomenológico, de vinte famílias. A partir de entrevistas aprofundadas e um grupo de discussão, na hora de conceitualizar e caracterizar um bom professor, os pais valorizam sua dimensão humana, a qual deve ser complementada com sua dimensão profissional, com componentes afetivos que permitem se relacionar empaticamente com os outros e gerar confiança e apoio formativo na criação de seus filhos. Os pais procuram estar tranquilos com respeito ao presente e ao futuro de seus filhos e, para isso, precisam da presença de bons professores que equilibrem o humano e o profissional no ato de educar.

\section{Palavras-chave (Fonte: Tesauro da Unesco)}

Qualidade da educação; competências do professor; desempenho docente; avaliação da educação; avaliação de professores; familia educação. 
El presente estudio aborda la temática del buen profesor, desde la perspectiva de las familias, con el propósito de conocer cuáles son los atributos, comportamientos y características valorados por las familias al momento de caracterizar al buen profesor. Desde la aparición del Informe Mackenzie (1983) sobre los sistemas de excelencia escolar en el mundo, que mostró que los profesores hacen la diferencia en los logros de aprendizaje, ha existido una preocupación por este actor relevante, respecto de sus actuaciones y del impacto que produce en los estudiantes y sus aprendizajes, es decir, por la enorme influencia que tiene en la calidad de la educación. El concepto da cuenta de la presencia de factores externos e internos que interfieren en esta. Entre los factores externos encontramos las estructuras sociales, culturales, económicas, políticas educativas, por nombrar algunos. En cuanto a los segundos, remiten a los elementos que se encuentran dentro de la escuela, como infraestructura, liderazgo directivo, materiales educativos, tecnología y, por supuesto, a uno de los más relevantes y con mayor impacto en los aprendizajes escolares: los profesores y sus actuaciones.

Las tendencias actuales en educación proponen la articulación de algunas de estas variables y de distintos actores para la consecución de logros educativos y aprendizajes. La Organización de las Naciones Unidas para la Educación, la Ciencia y la Cultura (Unesco, 1990) indica que la familia, con su participación, aportes y miradas sobre los fenómenos escolares, adquiere un papel relevante en los procesos de calidad educativa. Sin embargo, esta, muy pocas veces ha sido tomada en cuenta por las políticas educativas, pese a que ellas tienen que ver con temas referidos a la formación de sus hijos y el futuro de ellos.

Es así como se analizan los buenos profesores como factor interno relevante e influyente en la calidad de la educación (Bransford, Darling y Lepage, 2005; Sahlberg, 2013). Aun así, no existe un consen- so sobre las características y habilidades que ellos deben presentar para ser valorados como tales en el siglo XXI (Friedrich, 2014). Lo que podemos encontrar son aproximaciones diversas a esta figura, que van desde la identificación de las características de los profesores que obtienen buenos resultados en pruebas estandarizadas, hasta docentes que marcan la vida de sus estudiantes positivamente.

En actualidad, la búsqueda creciente y sistemática por alcanzar buenos resultados académicos, demostrables en pruebas nacionales e internacionales, ha transformado muchas veces el sentido de educar (en lo que se refiere a formar personas más allá de lo instrumental) y ello ha promovido ciertas prácticas educativas en desmedro de otras. Chile no es ajeno a esta búsqueda por alcanzar y buscar resultados de aprendizaje demostrables, lo que ha significado cambios importantes en distintos ámbitos, como infraestructura y leyes, como la de Inclusión en la Educación Superior, por nombrar alguna.

En relación con el profesor y sus actuaciones, se han implementado mecanismos de evaluación, como la política nacional para mejorar la calidad docente, que busca reconocer a un buen maestro y asegurarse de sus competencias y de sus desempeños (Roa, 2017). Esta preocupación se refleja en iniciativas que van desde atraer a los mejores egresados de la educación secundaria para ser futuros profesores y el acento en la formación inicial docente, hasta crear la carrera docente y recompensar a maestros de calidad y líderes escolares.

Al sintetizar distintos estudios sobre el buen profesor (Chetty, Friedman y Rockoff, 2014; López, 2010; Hargreaves, 1999; Rosenshine y Stevens, 1986; Gordon, Kane y Steiger, 2006; Murillo, 2003) es posible identificar claramente la presencia de dos visiones: 1) desde la perspectiva del docente efectivo (Barber y Mourshed, 2008; Rivkin, Hanushek y Kain, 2005), entendido como el sujeto que es capaz de impactar y obtener buenos resultados académicos en sus estudiantes; y 2) desde la perspectiva del 
docente afectivo, quien a partir de sus aspectos socioemocionales influye en los estudiantes y afecta su presente y futuro (Day y Gu, 2012; Merellano, Almonacid, Moreno y Castro, 2016). La primera visión apunta principalmente a la búsqueda de buenos resultados académicos de los estudiantes, lo que se ha relacionado con la presencia de buenos profesores, efectivos y eficientes en el logro de aprendizajes. Es decir, son aquellos profesionales que con sus comportamientos y actuaciones académicas son capaces de impactar significativamente en los resultados y aprendizajes de sus estudiantes (Winters, 2012).

El fundamento de esta posición que mira por los buenos resultados académicos está en el paradigma proceso-producto. Aquí, el acto de educar se asocia con resultados académicos observables y medibles y es llevado a cabo por un docente que, al lograr esas metas, se clasifica como un docente eficaz. Un buen docente sería entonces quien afecta positivamente en el rendimiento académico de los estudiantes, en desmedro de otras variables (Barber y Mourshed, 2008; Rivkin et al., 2005; Kukla, 2009; Radel, Sarrazin, Legrain y Wild, 2010).

Las actividades de enseñanza-aprendizaje efectivas en el aula, según Murillo (2003), se caracterizan por planificar la clase, generar un ambiente de disciplina, conocer la situación de los estudiantes respecto del avance en el proceso de aprendizaje, preocuparse de que estos se motiven, aprendan, expresen y encuentren sentido al conocimiento que se busca entregar, y llevar a cabo una retroalimentación (Acevedo, Valenti y Aguiñaga, 2017). Lo que persigue esta mirada es "conocer los rasgos, actitudes y comportamientos de los docentes que parecían tener un efecto beneficioso en el desarrollo de los alumnos" (Murillo, 2007, p. 29).

No hay que olvidar que, además de esta visión más instrumental del profesor y del acto de educar, es posible identificar la existencia de una larga contribución al concepto buen profesor, desde la pers- pectiva de desarrollo humano y social, fundada en los atributos relacionales, "concepto que engloba las relaciones sociales que circulan en el contexto de enseñanza-aprendizaje e incluye la conexión afectiva entre profesor y estudiante" (Kriewaldt, 2015, p. 85). Para esta mirada, el buen profesor se asocia a la influencia que tiene en la transformación de las personas. Una búsqueda menos instrumental y más asociada al rol del profesor como educador y formador de ciudadanos (Maturana, 1995). El buen profesor es quien impacta en otros ámbitos, más allá del aprendizaje académico medible en pruebas estandarizadas. Chetty et al. (2014) indican que los buenos maestros afectan las trayectorias de vida de los estudiantes

Entre esas características humanas de estos buenos profesores se encuentran: la moral (López, 2003), lo afectivo (Freire, 2004; López, 2010; Day, 2006), el tipo de interacciones que conforman con otros (Freire, 1990; Chomsky, 2001; Garcallo, Sánchez, Ros y Ferreras, 2010; Merellano, Almonacid, Moreno y Castro, 2016), el fuerte compromiso con su quehacer (Freire, 1990; Darling-Hammond, 2001); ser capaz de influir en la resiliencia de sus estudiantes (Day y Gu, 2012), en su autorrealización (Rogers, 2004) y en la identidad de sus aprendices (Jennings y Greenberg, 2009).

En síntesis, ambas miradas se hacen presentes cuando se investiga sobre lo que se considera que han de ser los buenos profesores. Las dos tienen que ver con aprendizajes y cambios. Una se relaciona con los atributos ligados a la profesión docente, más técnica e instrumental, y se produce en la relación profesor-estudiante en términos de logros académicos. La otra, relacionada con aprendizajes de elementos más subjetivos, de largo aliento y para la vida, se da en la relación profesor-estudiante, en términos de coherencia de los buenos profesores, en la comunicación, el afecto y compromiso, por nombrar algunos valores, que lo hacen un sujeto que marca de por vida, positivamente, a sus estudiantes. 


\section{Un buen profesor para los padres y apoderados}

La escuela, según Vain (2009), surge en la modernidad a partir de un pacto en el que las familias entregaron al Estado la responsabilidad de educar a sus hijos. En ese acuerdo, el Estado y sus técnicos asumieron completamente las decisiones sobre cómo hacerlo, qué se debe aprender, cómo enseñar y cuál currículo de formación de niños y jóvenes es necesario implementar. Sin embargo, esta manera de hacerlo no ha sido capaz de disminuir las desigualdades sociales ni de aumentar los logros académicos en los sectores socioeconómicos bajos, como tampoco ha contribuido a aumentar el desarrollo humano.

La complejidad del fenómeno educativo y la evidencia científica han demostrado que es necesario reconocer la importancia de otros agentes en el proceso de educar y formar. Se ha destacado en el último tiempo el impacto de las familias en los logros escolares cuando se involucran en el proceso de enseñanza y aprendizaje (Unicef, 2004). Dicho involucramiento, según Sánchez, Reyes y Villarroel (2016), lo hacen:

a. En comunicación con la escuela y con apoyo de los aprendizajes del aula, lo que incluye aspectos como la asistencia a la escuela, la comunicación con los maestros, el conocimiento del funcionamiento de la escuela, la comunicación con los hijos y la ayuda en la realización de tareas.

b. Con provisión de recursos educativos, tales como la inversión de tiempo en instrucción fuera del aula y actividades que enriquecen los aprendizajes de los estudiantes.

c. Con una perspectiva que aborda la participación en un sentido democrático, ligado al grado en que los padres, madres y apoderados participan en la toma de decisiones dentro de un establecimiento.
Se hace necesario, además de estas maneras de involucramiento, incluir en los debates educativos la voz de estos actores, en otros niveles, que puedan influir en las políticas, las prácticas y los aprendizajes de las escuelas (Vaillant, 2008). Promover las perspectivas de los padres y apoderados sobre la educación de sus hijos, en general, y sobre los profesores, en particular, es un tema relevante, ya que "la percepción que las familias tienen del trabajo de los profesores y la valoración que sobre él manifiestan son factores que influyen decisivamente en las relaciones que se establecen entre unos y otros" (Marchesi y Pérez, 2005, p. 16).

Los sistemas educativos y la sociedad se han dado cuenta de los efectos positivos de la participación de los padres en la educación de los hijos, aunque de manera incipiente y desorganizada. Esos actores cohabitan y son partícipes de la construcción de ese buen docente, ya que los maestros actúan como modelos junto con los padres (Raufelder, 2007).

Respecto de lo que valoran los padres en los buenos docentes, Román (2010, p. 8) indicó que buen docente es quien "se preocupa por los diferentes estilos de aprendizaje de sus estudiantes, logra motivarlos e interesarlos en el aprender, y mantiene un buen trato con ellos y las familias. Como cuarta prioridad aparece su capacidad para lograr buenos resultados o rendimiento con los alumnos, junto con su interés por perfeccionar y actualizar sus conocimientos".

Lo que da cuenta este estudio es que el tema de rendimiento no es relevante para estos padres y apoderados, sino que sus valoraciones estaban asociadas más bien a los estilos, las motivaciones y el trato otorgado por los docentes a sus hijos.

En estudios más recientes realizados en Chile, las familias valoran las capacidades, dedicación y forma de interacción que presentan los profesores con sus hijos (GTD-PREAL, 2009). Las expectativas de estos se pueden resumir en la búsqueda de que sus hijos aprendan de la mano de buenos docentes (Ló- 
pez, 2003), quienes poseen ciertas características a la hora de llevar cabo sus funciones.

\section{Diseño metodológico}

Este estudio se realizó con una metodología cualitativa, en la cual existe la posibilidad de aproximarnos aproximarse a las relaciones que se presentan entre la información expresada y el contexto de donde esta se obtiene, permitiendo la comprensión de las significaciones producidas. (Guba y Lincon, 2002). Utilizando un enfoque fenomenológico, sobre un estudio de caso, se estudió la realidad del clima de relaciones interpersonales en el aula, a través de la percepción subjetiva de los actores educativos (Sandín, 2003).

Las interpretaciones que los actores hacen de la realidad son construcciones intangibles, fundamentadas en la realidad de sus experiencias sociales particulares, que no operan con criterios tácitos de verdad (Guba y Lincon, 2002). En la presente investigación, el caso lo constituyen las familias de los estudiantes de una escuela de la comuna Pedro Aguirre Cerda, en Santiago de Chile. Las familias, aquí, pertenecen al nivel socioeconómico bajo, con altos niveles de vulnerabilidad, y bajos niveles de estudios.

De acuerdo con los objetivos, el caso se construyó a partir de los siguientes criterios: padres y apoderados de ambos sexos, de ambas jornadas escolares, familias de estudiantes de 10 a 11 años y 12 a 13, familias de hijos que proporcionalmente estuvieran bajo el promedio, en el promedio y sobre el promedio del curso y que tuvieran interés en participar de este estudio. De esta manera el caso quedó compuesto por 20 familias que se caracterizan en la Tabla 1.

Los instrumentos aplicados para investigar las percepciones de estos actores fueron: entrevistas en profundidad y un grupo focal de discusión, que permitió confrontar la información individual con la grupal, poniendo en contacto diferentes perspectivas, experiencias y puntos de vista (Valles, 2007).
Tabla 1. Caracterización general del caso

\begin{tabular}{|c|c|c|c|}
\hline ENTREVISTADOS & Sexo & Curso & Promedio hijo (a) \\
\hline Informante 1 & $\mathrm{~F}$ & $7 \mathrm{mo}$. & Sobre el promedio \\
\hline Informante 2 & $\mathrm{~F}$ & 8 vo. & Bajo el promedio \\
\hline Informante 3 & $\mathrm{~F}$ & 6to. & En el promedio \\
\hline Informante 4 & $M$ & 8 vo. & En el promedio \\
\hline Informante 5 & $\mathrm{~F}$ & 6to. & En el promedio \\
\hline Informante 6 & $\mathrm{~F}$ & 8 vo. & Sobre el promedio \\
\hline Informante 7 & $\mathrm{~F}$ & $7 \mathrm{mo}$. & En el promedio \\
\hline Informante 8 & $\mathrm{~F}$ & 5to. & Sobre el promedio \\
\hline Informante 9 & $\mathrm{~F}$ & 6to. & Sobre el promedio \\
\hline Informante 10 & $\mathrm{~F}$ & 8 vo. & Bajo en el promedio \\
\hline Informante 11 & M & $7 \mathrm{mo}$. & Bajo en el promedio \\
\hline Informante 12 & $\mathrm{~F}$ & 6to. & En el promedio \\
\hline Informante 13 & $\mathrm{~F}$ & 5to. & Bajo el promedio \\
\hline Informante 14 & $\mathrm{~F}$ & 8vo. & En el promedio \\
\hline $\begin{array}{l}\text { GRUPO DE } \\
\text { DISCUSIÓN }\end{array}$ & Sexo & Curso & Promedio hijo (a) \\
\hline Informante 1 & $\mathrm{~F}$ & 8vo. & Sobre el promedio \\
\hline Informante 2 & $\mathrm{~F}$ & $8 \mathrm{vo}$ & Bajo el promedio \\
\hline Informante 3 & $\mathrm{~F}$ & 6to. & En el promedio \\
\hline Informante 4 & $\mathrm{~F}$ & 6to. & Bajo el promedio \\
\hline Informante 5 & $\mathrm{~F}$ & 5to. & En el promedio \\
\hline Informante 6 & $\mathrm{~F}$ & $8 \mathrm{vo}$ & Sobre el promedio \\
\hline
\end{tabular}

Fuente: elaboración propia.

En el análisis fenomenológico, se procedió a organizar la información, para lo cual se codificaron los registros comunicativos que expresaban sentidos compartidos de las familias sobre el fenómeno estudiado. A partir de esos significados comunes, se categorizaron las respuestas, conceptos o temas similares descubiertos por los investigadores en esta temática (Rubin y Rubin, 1995). Para ello se ocupó el software Weft ODA, que clasifica los documentos transcritos que luego se agrupan, hasta que se genera un concepto abarcador.

\section{Resultados}

Las categorías del significado de un buen profesor que emergieron de los discursos se sintetizan en la Tabla 2: 
Tabla 2. Significado de un buen profesor: categorías

\begin{tabular}{|c|c|c|c|}
\hline Categoria & Subcategoria & Elementos & Caracteristicas que presentan los buenos profesores \\
\hline \multirow{5}{*}{$\begin{array}{l}\text { Dimensión } \\
\text { humana }\end{array}$} & \multirow{2}{*}{$\begin{array}{l}\text { Comunicación } \\
\text { reciproca }\end{array}$} & $\begin{array}{l}\text { Conversaciones de } \\
\text { futuro }\end{array}$ & Motivan, aconsejan, con expectativas que ayudan \\
\hline & & Escuchar empático & Escuchan, ayudan, empáticos, comprensivos \\
\hline & $\begin{array}{l}\text { Emociones al } \\
\text { servicio del proceso }\end{array}$ & Regulación emocional & $\begin{array}{l}\text { Alegres, novedosos, motivados, con dominio } \\
\text { emocional, amenos }\end{array}$ \\
\hline & \multirow{2}{*}{ Ética que modela } & La consecuencia & Respetuosos, verdaderos, consecuentes \\
\hline & & El compromiso & Comprometidos, confiables, cómplices, acogen \\
\hline \multirow{2}{*}{$\begin{array}{l}\text { Dimensión } \\
\text { profesional }\end{array}$} & \multirow{2}{*}{ Eficacia docente } & Didáctica utilizada & $\begin{array}{l}\text { Explican bien, usan metodologías adecuadas, son } \\
\text { dedicados y pacientes }\end{array}$ \\
\hline & & Docente riguroso & $\begin{array}{l}\text { Planificados, eficaces, exigentes, responsables, } \\
\text { estrictos, presionan }\end{array}$ \\
\hline
\end{tabular}

Fuente: elaboración propia.

A continuación, se presentarán las categorías emanadas de la información recopilada y su discusión e interpretación. Una de ellas tiene que ver con la dimensión humana del profesor. Refiere a la valoración que hacen los padres de elementos relacionados con la personalidad y las capacidades para relacionarse, sobre la importancia de generar relaciones escolares basadas en el afecto y compromiso de estos con sus hijos, la que se demuestra en la vocación y en la empatía con la que asumen su labor. Esto genera en los padres la confianza necesaria en lo que pueden lograr sus hijos en términos humanos y de valores.

Por otro lado, en cuanto a la dimensión profesional del buen profesor, tiene que ver con las valoraciones que hacen los padres de los elementos técnicos de estos docentes, su carácter profesional, el que al realizar sus clases demuestra su idoneidad, con competencias para enseñar de manera adecuada. Esto les da a los padres la tranquilidad necesaria para enfrentar los procesos de enseñanza-aprendizaje de sus hijos.

\section{El buen profesor en su dimensión humana}

Los padres buscan que sus hijos sean valorados y reconocidos. Para ello es fundamental la presencia de un buen maestro que genere confianza. Este es visto como un ser humano capaz de acoger, brindar apoyo y comprometerse con sus hijos y sus procesos, siendo muchas veces la persona que cumple el rol de padre en este contexto social: "la profesora igual tiene que ser como una mamá, porque en el fondo uno, el profesor, esta con un niño que no está con su padre, a pesar que no es su hijo, pero ella debiera tomarlo desde esa forma" (E.19730-20207).

Los relatos de las familias tienen puntos de concordancia con el Marco para la Buena Enseñanza (Mineduc, 2003) que, en su Dimensión B, indica la importancia de la creación de un ambiente propicio para el aprendizaje, el cual depende, en gran medida, de componentes sociales y afectivos. Dicha dimensión establece que las habilidades involucradas en este dominio se demuestran principalmente en la existencia de un ambiente estimulante y un profundo compromiso del profesor con los aprendizajes y el desarrollo de sus estudiantes.

\section{Comunicación}

Esta valoración, del profesor y de sus actuaciones se relaciona con la capacidad de relacionarse con los alumnos, sus hijos. La cual se produce desde el lenguaje, en espacios conversacionales (hablar y escuchar), lo que trae tranquilidad a los padres por las relacionales establecidas. Los elementos que conforman esta subcategoría son: 
Conversaciones de fUtURo: Los buenos profesores son los que se comunican con los estudiantes y los motivan a alcanzar sus metas, buscar nuevos caminos y perseverar en dicha búsqueda: "Absolutamente, el respeto yo creo que siempre le está inculcándoles a los niños como una buena conducta, las formas como se tiene que comportar, y siempre incentivando el tema del estudio, que siempre hay que proyectarse lo ideal, él dice que siempre lo ideal es la universidad, pero o si no pero siempre estudiar algo más, no quedarse" (L-18200-18600). Con sus palabras esos docentes son capaces de mostrar, incentivar, pretendiendo, muchas veces de manera inconsciente, intervenir y modificar el curso de los hechos, presentes en las vidas de padres y estudiantes, en el contexto social que se desenvuelven.

Escuchar empátICO: Los buenos profesores son aquellos que son capaces de escuchar, y que se dan tiempo para entender lo que les sucede a ellos y a sus hijos. Esto les otorga espacios de tranquilidad y confianza en su labor: En el área humana es importante "que sea comprensivo con los niños, porque van pasando por distintas etapas y hay que entenderlo, porque uno va cambiando el chip constantemente, tiene que entenderlo, apoyarlos, porque hay muchos niños que a lo mejor tienen problemas en sus casas y vuelven acá y de repente estallan acá; entonces hay que entenderlos, y la comunicación entre el papá y el profesor es muy importante" (MR-38544251). Esta subcategoría, relacionada con aspectos comunicacionales, lleva implícita la importancia de los espacios conversacionales y el valor de la interacción de los buenos docentes con los estudiantes, ya que lo que buscan los padres es encontrar apoyo a las problemáticas que presentan sus hijos.

\section{Subcategoría: emociones al servicio del proceso}

Las emociones que presenta el profesor permiten a los padres reconocer una vocación en ellos, la que es percibida en las acciones realizadas por el docente en el encuentro con los apoderados y en lo que expresan sus hijos de sus profesores en la conversación cotidiana con los padres y apoderados. El elemento que forma parte de este ámbito es el de la regulación emocional.

ReGULACIÓN emocional: Los buenos profesores son capaces de regular sus emociones de acuerdo con los momentos que viven en el aula. Seriedad cuando es necesario y poner límites y entusiasmo cuando invitan a tomar atención. Crear espacios educativos entretenidos hace que se les valore como docentes novedosos y alegres. Los padres valoran al profesor que es capaz de transmitir, en el ejercicio de sus labores, a través de sus actos y sus formas, una actitud que refleja y representa un gusto por la actividad pedagógica y la posibilidad de que sus hijos aprendan. "Ser como bien dinámica en sus clases, porque [a] clases aburridas niños aburridos, niños que no aprenden" (M-10312-10390).

\section{Subcategoría: ética que modela}

Los padres revelan la importancia de que el profesor inculque valores y forme a sus hijos y de que esas acciones estén en consonancia con los valores de las familias. Además, estiman que tomen conciencia de sus acciones y lo que provocan en los demás. Una apoderada lo expresa: "Por lo mismo que yo te digo, que porque la enseñanza de la casa, los valores que uno le entregan, ellos lo perciben a los profesores, en otra persona en realidad, en este caso el profesor y ellos se sienten acogidos y ellos mismos si tienen problemas, saben a quién acudir, porque le dan más confianza" (MI-7396-7750). En los buenos profesores existe coherencia entre lo que dicen y lo que hacen, lo que genera confianza en los padres. Los elementos de esta categoría son:

LA CONSECUENCIA: En los buenos profesores existe coherencia entre lo que dicen y lo que hacen, lo que genera confianza en los padres: "siempre con la verdad por delante, les guste o no les guste, o sea que reciben críticas buenas y críticas malas y que si tienen algo en contra de nosotros que también lo digan" (Grupo de discusión-3248-3806). 
EL Compromiso: Se establecen relaciones cercanas impregnadas por lazos afectivos, basadas en el respeto y la comunicación mutua, lo que provoca la sensación de estar frente a un ser que cuida, protege y se preocupan por el bienestar de sus hijos: "los profesores son muy buenos, enseñan valores y otras cosas que en otros colegios se están perdiendo" (S-984-1129). Esta valoración de las familias es posible asociarla con la enorme crisis de confianza que tienen los chilenos. Market Opinion Research International (MORI, 2016) indica que en Chile desde hace 28 años existen los más bajos niveles de confianza interpersonal y que hoy $77 \%$ de los chilenos no confían en el otro. Los padres valoran este ámbito, ya que les permitiría confiar en los buenos profesores y en la labor de formar adecuadamente a sus hijos.

\section{El buen profesor en su dimensión profesional}

La valoración de elementos profesionales en un buen profesor les da tranquilidad a los padres, ya que sus hijos van a aprender lo necesario, en términos de contenidos y materias propias de los cursos en los que se desenvuelven. Esto ayudará a sus hijos en su futuro. Este buen docente asume el proceso de enseñanza y aprendizaje de manera efectiva, con estrategias y metodologías adecuadas que le permitan cumplir con los objetivos y aprendizajes perseguidos. Los relatos de las familias tienen ciertos puntos de concordancia con lo expresado por el Marco para la Buena Enseñanza, que en su Dimensión A promueve la preparación para la enseñanza y se refiere "tanto a la disciplina que enseña el profesor o profesora, como a los principios y competencias pedagógicas necesarios para organizar el proceso de enseñanza, en la perspectiva de comprometer a todos sus estudiantes con los aprendizajes, dentro de las particularidades específicas del contexto en que dicho proceso ocurre" (2003). Esta dimensión está siendo cubierta de manera más o menos satisfactoria por los centros de formación inicial de profesores y en las evaluaciones docentes. Un estudio de Bruns y Luque de 2015 indica que la calidad de los profesores de Suramérica se ve comprometida por un pobre manejo de los contenidos académicos y por prácticas ineficaces en el aula.

\section{Subcategoría: eficacia docente}

Los padres valoran que el docente logre los resultados esperados en términos académicos y para ello este realiza una serie de acciones planificadas y destinadas al logro de dichos resultados académicos: "A mí me encanta como trabaja la Verónica; creo que ella es una buscadora de nuevas metodologías, nuevas formar de enseñar. ¡Eh! es estricta, sí; a lo mejor los chiquillos como que les molesta, pero yo creo que el objetivo lo logra: que es enseñar, y a ella le gusta lo que hace" (R-9279-9551). Esta subcategoría presenta dos elementos: didáctica utilizada, rigurosidad docente:

DidÁctICA UTILIZADA: La valoración de los padres se produce por la sensación de un trabajo racional, planificado, que responde a ciertas lógicas. En la búsqueda del logro de los aprendizajes esperados, buscan apoyo, con dedicación y paciencia, sin hacer diferencias: "yo pido que un profesor sepa enseñar al niño, que tenga la paciencia porque lo primordial para mí es la paciencia que tienen que tener y que de repente se sientan como las profesoras como mamá" (P-20254-20488).

RigurosidAD DOCENTE: Un buen docente es aquel que ocupa el tiempo de la clase de manera efectiva, da trabajos, es riguroso en la revisión y exige cumplimiento de los compromisos escolares, con estándares altos de desempeño. "Oue sea estricto, que las horas de clases sean para estudiar, que les pueda explicar bien la materia y que todos los niños entiendan. No que explique y el que entendió, entendió, y vamos con otra materia" (M-3425-3627).

En esa valoración de trabajo del docente se produce por la sensación, por parte de los padres, de la existencia de un trabajo racional, planificado, 
que responde a ciertas lógicas, y que el trabajo que realiza es acorde con esa finalidad perseguida y estructurada previamente. Esto les permite a los padres sentir seguridad respecto del presente y futuro de sus hijos: "que no sean como fue uno, que sean superiores, es siempre el consejo que les doy a mis hijos" (E-14652-15092).

Esta subcategoría, que tiene que ver con la eficacia del docente, resalta la importancia de la didáctica y la rigurosidad del docente con la formación académica de sus hijos. Esta valoración está dada por la necesidad de que los hijos salgan adelante, se desarrollen en el futuro y logren sus metas. Para ello es necesario que aprendan ciertos contenidos necesarios para esa experiencia futura y para lo cual necesitan buenos profesores.

\section{Discusión}

El objetivo del presente estudio fue comprender qué significa ser un "buen profesor" para los padres y apoderados en un colegio vulnerable de Santiago, a partir de la identificación y descripción de las características de este actor educativo. A partir del análisis de los discursos es posible indicar que, a la hora de conceptualizar y caracterizar a un buen docente, los padres valoran la dimensión humana de este, con componentes afectivos que le permiten relacionarse empáticamente con los otros y generar confianza y apoyo formativo en la crianza de los hijos. Tal dimensión debe ser complementada por la profesional, con componentes didácticos que tendrían como finalidad permitir a los hijos lograr los aprendizajes esperados. Es decir, un buen profesor debe ser capaz de equilibrar esas dimensiones en su profesión y en el acto de educar (Raufelder, Nitsche, Breitmeyer y Kebler, 2016).

Los padres y apoderados buscan estar tranquilos y seguros acerca de la educación que están recibiendo sus hijos y para lograrlo necesitan un buen profesor. Este posee características sociales que le permiten relacionarse adecuadamente con los estu- diantes y motivar e influir en su conducta, de modo que sea consecuente con sus acciones. Estas valoraciones responden a la necesidad de sentir apoyo y seguridad en las diversas situaciones de vulnerabilidad, cotidianas y futuras, de su entorno familiar y social, las que causan incertidumbre y temor.

Estos hallazgos apoyan investigaciones anteriores que muestran que el buen profesor se construye en relaciones de diálogo, respeto y compromiso con el alumno (Freire, 1990; Hargreaves, 1999; López, Delessert y Pérez, 2011), siendo capaz de crear y mantener un entorno de apoyo emocional (Luckner y Pianta, 2011), que refuerza la motivación y el esfuerzo (Fredriksen y Rhodes, 2004). La alegría y el gusto percibido en esos profesores influyen en la predisposición de los estudiantes y en la experiencia de aprender (Birch y Ladd, 1997).

Las familias desean que sus hijos "sean alguien en la vida", para lo cual necesitan aprender adecuadamente las materias y contenidos planteados en la escuela. La escuela y los buenos profesores deben brindar las herramientas necesarias para que sus hijos puedan enfrentar la vida. Los logros académicos dependen de la presencia de un buen profesor, que posea una didáctica adecuada y sea riguroso y exigente cuando ejerce su labor pedagógica.

Esta mirada coincide con el paradigma procesoproducto y las concepciones elaboradas por autores como Bloom (1981), quien otorga un papel importantey relevante al empleo de estrategias de instrucción en los logros y aprendizajes. Para Murillo (2007) la eficacia escolar comporta una enseñanza adecuada, lo que incluye que los buenos docentes preparan sus clases, con unidades didácticas estructuradas, explican con claridad y evalúan los progresos.

En conclusión, de las narrativas de los padres y apoderados surgieron diversos conceptos respecto de lo que ellos consideran como buenos profesores, hallazgos que pueden ser utilizados como puntos de partida para mejorar la relación profesor-alumno 
y profesores-padres en las escuelas y confirmar gran parte de lo que podemos encontrar en la teoría referida al tema.

\section{Proyección de los resultados}

Es necesario articular el valor que asignan los padres a las actuaciones de los docentes con las políticas públicas en esta materia. En esa línea, los padres dan un valor importante a las relaciones positivas de empatía, cercanía, compromiso, alegría que se dan en la escuela, las cuales influyen en los ambientes escolares y en la predisposición a aprender. Desde ahí la necesidad de incorporar el componente humano en la formación del futuro docente y no solo enfatizar en los aspectos profesionales y técnicos.
Investigar y profundizar en los conocimientos sobre qué es ser un buen profesor y las valoraciones acerca de este actor permitiría abrir la reflexión a los componentes que valoramos como sociedad y también generar nuevos espacios para la formación de profesionales de la educación que respondan a los requerimientos de las familias. Con ello se hace más significativo el proceso educativo, ya que este se nutre de las miradas de diferentes actores, con sus necesidades. Consideramos que esto es posible, principalmente, por los enormes desafíos que tenemos como sociedad, en términos humanos, relacionales, de respeto, a los cuales no ha podido responder la educación actual.

\section{Referencias}

Acevedo, C., Valenti, G.y Aguiñaga, E. (2017). Gestión institucional, involucramiento docentey de padres de familia en escuelas públicas de México.Calidad en la Educación, 46,53-95.DOI: 10.4067/So718-45652017000100053

Barber, M. y Mourshed, M. (2008). Cómo hicieron los sistemas educativos con mejor desempeño del mundo para alcanzar sus objetivos. Preal.41,1-48. Recuperado de http://www.oei.es/pdfs/documento_preal41.pdf

Birch, S. y Ladd, G. (1997). The teacher-child relationship and children's early school adjustment. Journal of School Psychology, 35, 61-79. DOI: 10.1016/So022-4405(96)00029-5

Bloom, B. (1981). All our children learning. Nueva York: McGraw-Hill.

Bransford, J., Darling-Hammond, L.y Lepage, P. (2005). What teachers should learn and be able to do. En L. DarlingHammond y. Bransford (eds.), Preparing teachers for a changing world (pp.1-39). San Francisco: Jossey Bass.

Bruns, B. y Luque, J. (2015). Great teachers: How to raise student learning in Latin America and the Caribbean. Washington: Banco Mundial.

Chetty, R., Friedman, J. y Rockoff, J. (2014). Measuring the impacts of teachers I: Evaluating bias in teacher valueadded estimates. American Economic Review, 104(9), 2593-2632. DOI: 10.3386/w19423

Chomsky, N. (2001). La (Des)educación. Barcelona: Crítica.

Day, C. (2006). Pasión por enseñar. Madrid: Narcea.

Day, C. y Gu, O. (2012). Profesores: vidas nuevas, verdades antiguas. Madrid: Narcea. 
Darling-Hammond, L. (2001). El derecho de aprender. Crear buenas escuelas para todos. Barcelona: Ariel.

Friedrich, D. (2014). "We brought it upon ourselves": University-based teacher education and the emergence of boot-camp-style routes to teacher certification. Education Policy Analysis, 22, 2. DOI: 10.14507/epaa. v22n2.2014

Fredriksen, K. y Rhodes, J. (2004). The role of teacher relationships in the lives of students. New Directions for Youth Development, 103, 45-54. DOI:10.1002/yd.90

Freire, P. (1990). La naturaleza politica de la educación. Cultura, poder y liberación. Barcelona: Paidós.

Freire, P. (2004). Cartas a quien pretende enseñar. Buenos Aires: Siglo XXI.

Garcallo, B., Sánchez, F., Ros, C. y Ferreras, A. (2010). Estilos docentes de los profesores universitarios. La percepción de los alumnos de los buenos profesores. Revista Iberoamericana de Educación, 51(4), 1-16. Recuperado de https://rieoei.org/RIE/article/view/1826

Gordon, R., Kane, T.y Steiger, D. (2006). Identifying effective teachers using performance on the job. Washington: Brookings Institution.

GTD-PREAL. (2009). Así opinan los padres sobre los docentes. Boletín Electrónico GTD-PREAL. 36, 1-50. Recuperado de http://www.preal.org/Grupo.asp?ld_Grupo=5\&ld_Seccion=36

Guba, E. y Lincon, Y. (2002). Por los rincones. Antología de métodos cualitativos en la investigación social. En V. A. Deuman y J. A. Haro (eds.), Paradigmas en competencia en la investigación cualitativa. (pp. 113-145). Hermosillo: El Colegio de Sonora.

Hargreaves, A. (1999). Cuatro edades del profesionalismo y aprendizaje profesional. En B. Avalos y M. E. Nordenfly cht (eds.), La formación de profesores. Perspectiva y experiencias. Santiago: Santillana.

Jennings, P. y Greenberg, M. (2009). The prosocial classroom: Teacher social and emotional competence in relation to student and classroom outcomes. Review of Educational Research, 79(1), 491-525. DOI: 10.3102/0034654308325693

Kriewaldt, J. (2015). Strengthening learners' perspectives in professional standards to restore relationality as central to teaching. Australian Journal of Teacher Education, 40(8). Recuperado de http://ro.ecu.edu.au/ cgi/viewcontent.cgi?article $=2490 \&$ context $=$ ajte

Kukla-Acevedo, S. (2009). Do teacher characteristics matter? New results on the effects of teacher preparation on student achievement. Economics of Education Review, 28, 49-57. DOI:10.1016/j.econedurev.2007.10.007

López, R., Delessert, E.y Pérez, S. (2011). Coaching para profesores. Perfil del profesional del siglo XXI. Chile: Editorial Jurídica de Santiago.

López, S. (2010). Las complejidades emergentes en las historias de vida de los "buenos profesores". Polis, 9(25), 255-267. Recuperado de http://journals.openedition.org/polis/465 
López, S. (2003). Construcción sociocultural de la profesionalidad docente: estudio de casos de profesores comprometidos con un proyecto educativo. (Tesis Doctoral), Departamento de Didáctica, Universitat de Valencia, España.

Luckner, A. y Pianta, R. (2011). Teacher student interactions in fifth grade classrooms: Relations with children's peer behavior.Journal of Applied Developmental Psychology,32(5),257-266.DOI:10.1016/j.appdev.2011.02.010

Mackenzie, D. (1983). Research for school improvement: An appraisal of some recent trends. Education Research, 12(4), 5-17. DOI: 10.3102/0013189X012004005

Marchesi, A. y Pérez, E. (2005). Opinión de las familias sobre la calidad de la educación. Madrid: Centro de Innovación Educativa - Fuhem.

Maturana, H. (1995). Formación humana y capacitación. Santiago: Unicef/Dolmen.

Merellano, E., Almonacid, A., Moreno, A. y Castro C. (2016). Buenos docentes universitarios: ¿Qué dicen los estudiantes? Educação e Pesquisa, 42(4), 937-952. Recuperado de https://www.redaly c.org/articulo. oa?id=29848830005

Mineduc (2003). Marco para la Buena Enseñanza. Santiago de Chile.

MORI-CERC (2017). Barómetro de la política en Chile. MORI. Recuperado de: http://morichile.cl/wp-content/ uploads/2017/10/Informe-de-prensa-barometro-pol-set-2017.pdf

Murillo, F. (2003). El movimiento de investigación de eficacia escolar. Bogotá: Convenio Andrés Bello - Centro de Investigación y Documentación Educativa.

Murillo, F. (2007). Investigación iberoamericana sobre eficacia escolar. Bogotá: Convenio Andrés Bello.

Radel, R., Sarrazin, P., Legrain, P. y Wild, C. (2010). Social contagion of motivation between teacher and student: Analyzing underlying processes. Journal of Educational Psychology, 102, 577-587. DOI: 10.1037/a0019051

Raufelder, D. (2007). Von Machtspielen zu Sympathiegesten. Marburgo:Tectum.

Raufelder, D., Nitsche, L., Breitmeyer, S. y Kebler, S. (2016). Students' perception of "good" and "bad" teachersResults of a qualitative thematic analysis with German adolescents. International Journal of Educational Research, 75, 31-44. DOI: 10.1016/j.jjer.2015.11.004

Rivkin, S., Hanushek, E., y Kain, J. (2005). Teachers, schools and academic achievement. Econometrica, 73, 417-458. Recuperado de https://econ.ucsb.edu/ jon/Econ230C/HanushekRivkin.pdf

Roa-Tampe, K. A. (2017). La evaluación docente bajo la óptica del desarrollo profesional: el caso chileno. Educación y Educadores, 20(1), 41-61. DOI: 10.5294/edu.2017.20.1.3

Román, M. (2010). La voz ausente de estudiantes y padres en la evaluación del desempeño docente. Preal. 49, 1-30. Recuperado de:www.cide.cl/documentos/preal_49_MR.pdf 
Rogers, C. (2004). Grupos de encuentro. Argentina: Amorrortu.

Rosenshine, B. y Stevens, R. (1986). Teaching functions. En M. C. Wittrock (ed.), Handbook of Research on Teaching (3 ed., pp. 376-391). Nueva York: Macmillan.

Rubin, H.y Rubin, I. (1995). Qualitative interviewing: the art of hearing data. Londres: Sage.

Sahlberg, P. (2013). El cambio educativo de Finlandia. ¿Qué puede aprender el mundo? Buenos Aires: Paidós.

Sandín, M. (2003). Investigación cualitativa en educación. Fundamentos y tradiciones. Madrid: McGraw-Hill.

Sánchez, A., Reyes, F.y Villarroel, V. (2016). Participación y expectativas de los padres sobre la educación de sus hijos en una escuela pública. Estudios Pedagógicos, 42(3), 347-367. DOI:10.4067/S0718-07052016000400019

Unesco (2007). Educación de calidad para todos. Un asunto de derechos humanos. Santiago de Chile: Unesco-Prelac.

Unesco (1990). Declaración Mundial sobre educación para todos. Jomtien, Tailandia.

Unicef (2004). Contactos: Boletín Informativo para Centros de Padres No. 9. Santiago de Chile: Unicef - Cide.

Vain, P. D. (2009). Escuela, Estado y familia. Un pacto por redefinir. Educación, Lenguaje y Sociedad, 6, 329-344. Recuperado de http://www.biblioteca.unlpam.edu.ar/pubpdf/ieles/no6a17vain.pdf

Vaillant, D. (2008). Algunos marcos referenciales en la evaluación del desempeño docente. Revista Iberoamericana Evaluación Educativa, 1(2), 7-22. Recuperado de https://revistas.uam.es/index.php/riee/article/ view/4663

Valles, M. (2007). Técnicas cualitativas de investigación. Madrid: Sintesis.

Winters, M. (2012). Teachers matter. Lanham, MD: Rowman \& Littlefield. 